\title{
Performance Optimization of Self-organizing Bioparticles for Multi-target Detection and Gravitation Problems
}

\author{
Jian Yang ${ }^{1}$, Satoru Iwasaki ${ }^{1}$, Anthony O. Abraham ${ }^{2}$, \\ Juan Lorenzo Hagad ${ }^{1}$, Takuya Obuchi ${ }^{1}$, Tadashi Nakano ${ }^{2,3}$ \\ ${ }^{1}$ Graduate School of Information Science and Technology, Osaka University, Japan \\ ${ }^{2}$ Graduate School of Frontier Biosciences, Osaka University, Japan \\ ${ }^{3}$ Institute for Academic Initiatives, Osaka University, Japan \\ Email: yangjian@ist.osaka-u.ac.jp, satoru.iwasaki@ist.osaka-u.ac.jp \\ abraham@fbs.osaka-u.ac.jp, hagad@ai.sanken.osaka-u.ac.jp \\ obuchi.takuya@ist.osaka-u.ac.jp, tadasi.nakano@fbs.osaka-u.ac.jp
}

\begin{abstract}
This paper investigates the performance of self-organizing bioparticles for multi-target detection and gravitation problems. This paper describes three approaches to multi-target detection and gravitation problems in which bioparticles coordinate their behavior by secreting (1) attractants, (2) repellents or (3) both attractants and repellents. We first define the behavior of bioparticles in these approaches by using differential equations. We then show numerical results to understand the basic behavior of bioparticles in each approach and to demonstrate optimized performance for multi-target detection and gravitation problems.
\end{abstract}

\section{CCS Concepts}

-Applied computing $\rightarrow$ Health care information systems; $\bullet$ Hardware $\rightarrow$ Biology-related information processing;

\section{Keywords}

\section{INTRODUCTION}

Swarm intelligence is an emergent property that arises from a group of locally interacting individuals [1]. It is often observed in self-organized "intelligent" systems where autonomy, emergence and distributed functions replace control, pre-programming and centralization. Examples of such systems range from bacterial colonies [2] to insect colonies [1], fish schools [8] and bird flocks [4], which demonstrate intelligent behaviors including collective foraging, nest building and pattern formation (e.g., biofilm formation by bacteria).

Inspired by how a group of individuals in nature exhibit such intelligent behavior, this paper investigates the design of self-organizing bioparticles for nanomedical applications. Here we define the rules by which bioparticles interact in order to meet the application goals. In particular, we focus

Permission to make digital or hard copies of all or part of this work for personal or classroom use is granted without fee provided that copies are not made or distributed for profit or commercial advantage and that copies bear this notice and the full citation on the first page. To copy otherwise, to republish, to post on servers or to redistribute to lists, requires prior specific permission and/or a fee. BICT 2017, March 15-16, Hoboken, United States

ISBN 978-1-63190-148-5

DOI: 10.4108/eai.22-3-2017.152413

Copyright $\odot 2017$ EAI on a swarm of bioparticles that interact by secreting signaling molecules called attractants and repellents to detect and localize to targets (e.g. cancer cells) that may exist in the environment $[3,5,6,7,9]$.

The specific problem considered in this paper is referred to as the multi-target detection and gravitation problem [3] and illustrated in Fig. 1. Fig. 1(a) shows the spatially distributed targets (e.g. cancer cells) which concentrate in certain areas of the environment. The goal is to let bioparticles distribute themselves according to the target distribution utilizing attractants and repellents which the bioparticles themselves secrete. Fig. 1(b) assumes that the bioparticles are first injected at the top right corner in the environment. Bioparticles then release repellents to repel each other in order to efficiently search for targets in the environment. Bioparticles, upon detecting a target, release attractants to direct the motion of nearby bioparticles toward the target location. Overtime, bioparticles concentrate around target locations and distribute according to the target distribution spatially as shown in Fig. 1(c)-(g).

The multi-target detection and gravitation problem was investigated in [3]. In [3], bioparticles, upon detecting targets, release both attractants and repellents to induce gravitational forces toward target locations. In this paper, we examine three modified approaches by which bioparticles coordinate their behavior through secreting (1) attractants, (2) repellents or (3) both attractants and repellents. We examine the basic behavior of bioparticles in these approaches and evaluate the performance.

The rest of the paper is organized as follows. Section 2 describes three approaches (i.e., attractant-based, repellentbased and combined approaches) to the multi-target detection and gravitation problem, and the mathematical models that define the behavior of bioparticles in each approach. In Section 3, we show numerical results obtained using the mathematical models to understand the basic behavior of bioparticles and to demonstrate optimized performance. Finally, Section 4 summarizes the work described in this paper and discusses future challenges.

\section{MODEL EQUATIONS}

In this paper, we develop a continuous-space model of multi-target detection and gravitation problems where bioparticles, targets, attractants and repellents are all represented by concentration. For simplicity, our model focuses on a one- 


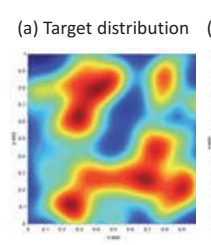

(e) Time $=30$

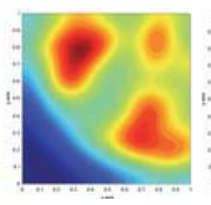

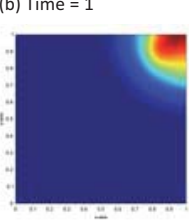

(f) Time $=40$

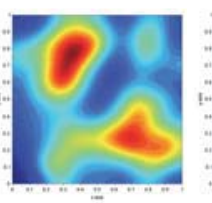

(c) Time $=10$

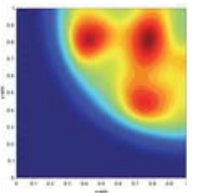

(g) Time $=80$

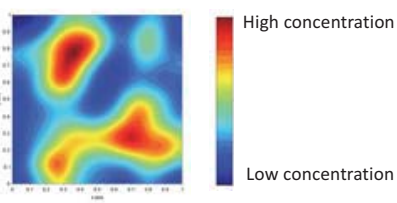

(d) Time $=20$
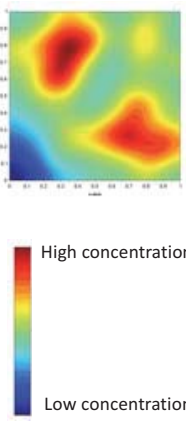

Figure 1: Multitarget detection and gravitation problem [3]

\begin{tabular}{l|l} 
Symbol & Description \\
\hline \hline$T(x)$ & Target concentration \\
$C_{b}(x, t)$ & Bioparticles concentration \\
$C_{a}(x, t)$ & Attractant concentration \\
$C_{r}(x, t)$ & Repellent concentration \\
$D_{b}$ & Diffusion coefficient of a bioparticle \\
$D_{a}$ & Diffusion coefficient of attractant \\
$D_{r}$ & Diffusion coefficient of repellent \\
$V_{a}$ & Attraction coefficient \\
$V_{r}$ & Repulsion coefficient \\
$g_{a}$ & Maximum attractant production rate \\
$g_{r}$ & Maximum repellent production rate \\
$K_{a}$ & Target concentration leading to half maximum \\
& attractant production rate \\
$K_{r}$ & Target concentration leading to half maximum \\
& repellent production rate \\
$k_{a}$ & Decay rate constant of attractant \\
$k_{r}$ & Decay rate constant of repellent
\end{tabular}

dimensional environment with immobile targets. However, these assumptions can be easily relaxed.

We use the notation in Table 1 to describe how $C_{b}(x, t)(=$ $\left.C_{b}\right), C_{a}(x, t)\left(=C_{a}\right)$ and $C_{r}(x, t)\left(=C_{r}\right)$ change with time. As will be described below in this section, we consider three approaches. Accordingly, we present three sets of partial differential equations (PDEs).

\subsection{Approach 1: use of attractants}

In the first approach, bioparticles release attractants at target locations to form attractant concentration gradients in the environment. Bioparticles move up the attractant concentration gradients and thus toward target locations. The set of PDEs describing this approach are given by

$$
\begin{aligned}
\frac{\partial C_{b}}{\partial t} & =D_{b} \frac{\partial^{2} C_{b}}{\partial x^{2}}-V_{a} \frac{\partial}{\partial x}\left(C_{b} \frac{\partial C_{a}}{\partial x}\right), \\
\frac{\partial C_{a}}{\partial t} & =D_{a} \frac{\partial^{2} C_{a}}{\partial x^{2}}+g_{a} \frac{T(x)}{T(x)+K a} C_{b}-k_{a} C_{a} .
\end{aligned}
$$

In the above set of PDEs, (1) describes the rate of change in bioparticle's concentration. This is based on the two types of diffusion through which bioparticles move in the environment: the random diffusion with the diffusion coefficient
$\left(D_{b}\right)$ and the directional diffusion based on the attractant concentration gradient with the attraction coefficient $\left(V_{a}\right)$. (2) describes the rate of change in attractant concentration; it is based on the diffusion coefficient $\left(D_{a}\right)$, the production rate $\left(g_{a} \frac{T(x)}{T(x)+K a} C_{b}\right)$ where bioparticles that are aware of targets are assumed to produce attractants, and the decay rate of attractants $\left(k_{a} C_{a}\right)$.

\subsection{Approach 2: Use of repellents}

In the second approach, bioparticles release repellents at non-target locations to form repellent concentration gradients in the environment. Bioparticles move down the repellent concentration gradients and thus toward target locations. The set of PDEs describing this approach are given by

$$
\begin{aligned}
\frac{\partial C_{b}}{\partial t} & =D_{b} \frac{\partial^{2} C_{b}}{\partial x^{2}}+V_{r} \frac{\partial}{\partial x}\left(C_{b} \frac{\partial C_{r}}{\partial x}\right), \\
\frac{\partial C_{r}}{\partial t} & =D_{r} \frac{\partial^{2} C_{r}}{\partial x^{2}}+g_{r} \frac{K r}{T(x)+K r} C_{b}-k_{r} C_{r} .
\end{aligned}
$$

The two equations (3) and (4) are similar to (1) and (2) in Approach 1, respectively. (3) describes the rate of change in bioparticle's concentration. It is based on the random diffusion with the diffusion coefficient $\left(D_{b}\right)$ and the directional diffusion based on the repellent concentration gradient with the repulsion coefficient $\left(V_{r}\right)$. (4) describes the rate of change in repellent concentration. It is based on the diffusion coefficient $\left(D_{r}\right)$, the production rate $\left(g_{r} \frac{K_{r}}{T(x)+K r} C_{r}\right)$ where bioparticles that are unaware of targets are assumed to produce repellents, and the decay rate of repellents $\left(k_{r} C_{r}\right)$.

\subsection{Approach 3: Combined use of attractants and repellents}

The third approach combines Approaches 1 and 2 in which bioparticles release attractants at target locations and repellents at non-target locations. The set of PDEs describing this approach are given by

$$
\begin{aligned}
\frac{\partial C_{b}}{\partial t} & =D_{b} \frac{\partial^{2} C_{b}}{\partial x^{2}}-\frac{\partial}{\partial x}\left[C_{b}\left(V_{a} \frac{\partial C_{a}}{\partial x}-V_{r} \frac{\partial C_{r}}{\partial x}\right)\right], \\
\frac{\partial C_{a}}{\partial t} & =D_{a} \frac{\partial^{2} C_{a}}{\partial x^{2}}+g_{a} \frac{T(x)}{T(x)+K a} C_{b}-k_{a} C_{a}, \\
\frac{\partial C_{r}}{\partial t} & =D_{r} \frac{\partial^{2} C_{r}}{\partial x^{2}}+g_{r} \frac{K r}{T(x)+K r} C_{b}-k_{r} C_{r} .
\end{aligned}
$$

In this set of PDEs, (5) describes the rate of change in bioparticle's concentration where the effects of attractants and repellents are combined. (6) and (7) describe the rate of change in attractant and repellent concentrations and identical to (2) and (4), respectively.

\section{PERFORMANCE EVALUATION}

\subsection{Performance Metric}

We use the Jensen-Shannon divergence (JSD) to measure the difference between bioparticle and target distributions. To use JSD, we use normalized concentrations of bioparticles and targets that satisfy $\int_{\alpha}^{\beta} C_{b}(x, t) d x=1$ (at any time $t$ ) and 
(A)

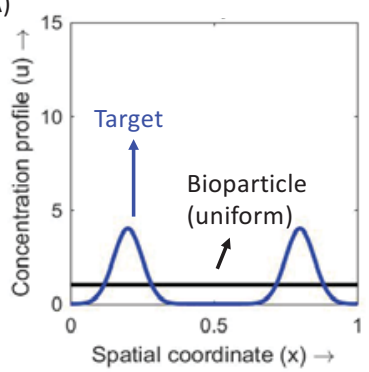

(B)

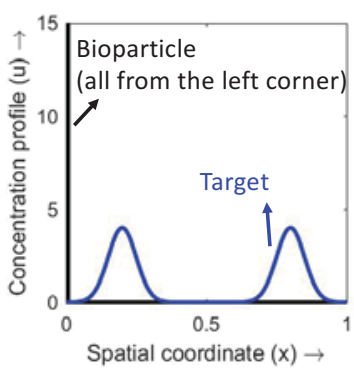

Figure 2: Target distribution (blue) and initial bioparticle distributions (black)

$$
\begin{aligned}
& \int_{\alpha}^{\beta} T(x) d x=1 . \\
& \operatorname{JSD}\left(T(x) \| C_{b}(x, t)\right)= \\
& \quad \frac{1}{2} D\left(C_{b}(x, t) \| M(x)\right)+\frac{1}{2} D(T(x) \| M(x)),
\end{aligned}
$$

where

$$
M(x)=\frac{C_{b}(x, t)+T(x)}{2},
$$

and $D(\cdot)$ is the Kullback-Leibler divergence defined as

$$
D(p(x) \| q(x))=\int_{\alpha}^{\beta} p(x) \log _{2} \frac{p(x)}{q(x)} d x .
$$

Note that the JSD above is a function of time $t$. Note also that it has the following properties.

- $\operatorname{JSD}(p(x) \| q(x))=\operatorname{JSD}(q(x) \| p(x))$.

- $0 \leq \operatorname{JSD}(p(x) \| q(x)) \leq 1$.

- $\operatorname{JSD}(p(x) \| q(x))=0$ when $p(x)=q(x)$.

\subsection{Configurations}

We assume that targets are concentrated around two different locations in the interval $I=[\alpha, \beta]$ : one location at $x=\mu_{1}$ and the other location at $x=\mu_{2}\left(\mu_{1} \neq \mu_{2}\right)$. Accordingly, we model $T(x)$ as $\mathcal{N}\left(\mu_{1}, \sigma^{2}\right)+\mathcal{N}\left(\mu_{2}, \sigma^{2}\right)$ normalized over $I$.

The boundaries of the interval are reflective under the assumption that bioparticles, attractants and repellents are confined in space (e.g., by vascular walls and perimeters of organs). By applying the Neumann boundary condition to $C_{b}, C_{a}$ and $C_{r}$, we have

$$
\frac{\partial C_{b}}{\partial x}=\frac{\partial C_{a}}{\partial x}=\frac{\partial C_{r}}{\partial x}=0 \text { at } x=\alpha \text { and } \beta .
$$

At time $t=0$, bioparticles are injected either at the leftcorner of the interval or distributed over the interval. Further, at $t=0$, attractants and repellents are not present in the interval. Therefore, the initial conditions become

$$
\begin{aligned}
& C_{b}(x, 0)=\delta(x-\alpha) \text { or } \frac{1}{\beta-\alpha}, \\
& C_{a}(x, 0)=C_{r}(x, 0)=0 .
\end{aligned}
$$

In numerical integrations of the model equations described in Section 2, we use $\alpha=0$ and $\beta=1$ (i.e., $|I|=1 \mathrm{~mm}$ ) and discretize space $(x)$ with $\Delta x=1 / 2000(\mathrm{~mm})$ and time $(t)$ with $\Delta t=0.01(\mathrm{sec})$. We also use $\mu_{1}=0.2, \mu_{2}=0.8$,

and $\sigma^{2}=0.0025$ (see Fig. 2 for the target distribution and initial bioparticle distributions). Other parameter values are arbitrary determined and shown in Table 2 .

Table 2: Default parameter values

\begin{tabular}{l|l|l} 
Symbol & Value & Unit \\
\hline \hline$D_{b}$ & 1 & $\mathrm{~mm}^{2} \cdot \mathrm{min}^{-1}$ \\
$D_{a}$ & 0.3 & $\mathrm{~mm}^{2} \cdot \mathrm{min}^{-1}$ \\
$D_{r}$ & 0.05 & $\mathrm{~mm}{ }^{2} \cdot \mathrm{min}^{-1}$ \\
$V_{a}$ & 0.1 & $\mathrm{~mm} \cdot \mathrm{min}^{-1}$ \\
$V_{r}$ & 0.1 & $\mathrm{~mm} \cdot \mathrm{min}^{-1}$ \\
$g_{a}$ & 1 & $\mu \mathrm{M} \cdot \mathrm{min}^{-1}$ \\
$g_{r}$ & 1 & $\mu \mathrm{M} \cdot \mathrm{min}^{-1}$ \\
$K_{a}$ & 1 & $\mu \mathrm{M}$ \\
$K_{r}$ & 1 & $\mu \mathrm{M}$ \\
$k_{a}$ & 0.1 & $\mathrm{~min}^{-1}$ \\
$k_{r}$ & 0.1 & $\mathrm{~min}^{-1}$
\end{tabular}

\subsection{Numerical Results}

In this section, we first perform numerical experiments using default parameter values to understand the basic behavior of bioparticles (in Sections 3.3.1 and 3.3.2). We then vary control parameters to optimize their target detection and gravitation performance (in Sections 3.3.3).

\subsubsection{Bioparticles injected uniformly}

Fig. 3 shows numerical results when bioparticles are uniformly injected in the interval at time $t=0$. Shown in this figure are JSD as a function of time (left) and the spatial distributions of attractants, repellents, bioparticles and targets at time $t=50$ (min) (right) when approaches 1,2 or 3 are used.

When Approach 1 is used, bioparticles release more attractants where the target concentration is higher (and they do not release repellents at all). In this case, JSD decreases first and then increases as shown in Fig. 3(A) (left). JSD decreases first because uniformly distributed bioparticles separate into two groups with each group concentrating at one of the two target locations. JSD however starts to increase at around time $t=10(\mathrm{~min})$. This is because each bioparticle group over-concentrates in a small interval near the respective target location as shown in Fig. 3(A) (right).

When Approach 2 is used, bioparticles release more repellents where the target concentration is lower (and they do not release attractants at all). In this case, JSD decreases and then remains low as shown in Fig. 3(B) (left). Unlike in the previous case where approach 1 is used, bioparticles in this case do not over-concentrate in a small interval; see Fig. 3(C) (right). Further, unlike in the previous case, we observe in this case that the bioparticle distribution (and hence JSD) oscillates with time and that the oscillation decays with time as indicated by JSD in Fig. 3(B) (left).

When Approach 3 is used, bioparticles release more attractants where the target concentration is higher. Bioparticles also release more repellents where the target concentration is lower. When approaches 1 and 2 are combined in this way, bioparticles achieve the best performance. The JSD decreases and remains low (see Fig. 3(C) (left)) and bioparticles form the fittest distribution to the target distribution among the three approaches as shown in Fig. 3(C) (right). 
(A) Uniform: Approach 1

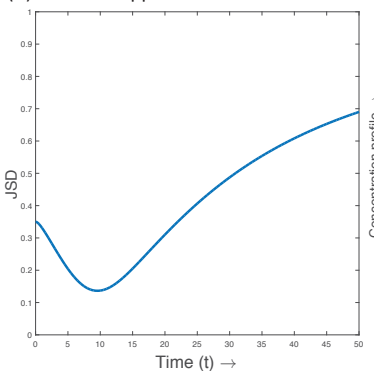

(B) Uniform: Approach 2

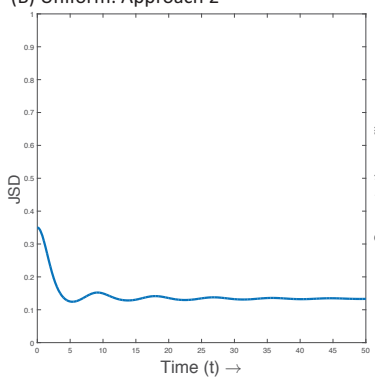

(C) Uniform: Approach 3
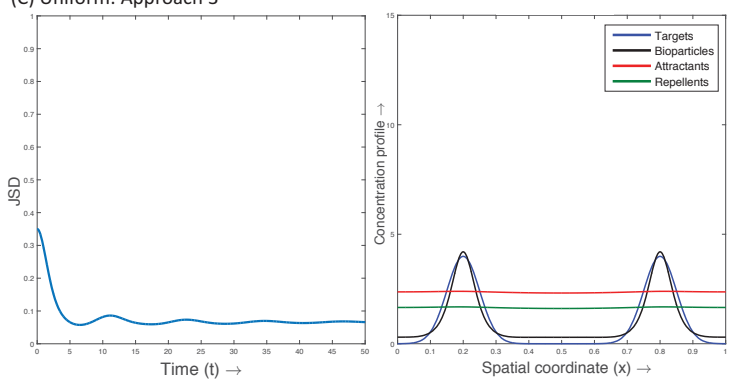

Figure 3: Experimental results. Bioparticles are injected uniformly.

\subsubsection{Bioparticles injected at a corner}

Fig. 4 shows numerical results when bioparticles are injected at the left corner of the interval at time $t=0$. Similar to Fig. 3, Fig. 4 shows JSD as a function of time (left) and the spatial distributions of attractants, repellents, bioparticles and targets at time $t=50$ (min) (right) when approaches 1, 2 or 3 are used.

When Approach 1 is used, JSD decreases first and then increases as shown in Fig. 4(A) (left). This is also observed in the previous case where approach 1 is used and bioparticles are injected uniformly (Fig. 3(A) (left)). Unlike the previous case, however, bioparticles in this case fail to separate into two groups (see Fig. 4(A) (left)). They concentrate at the nearest (left) target location, leaving the concentration of bioparticle at the other target location very low. This would be an undesirable behavior for drug delivery applications, since drug molecules are only delivered to one of the two target locations.

When Approach 2 is used, JSD and the bioparticle distribution continue to oscillate (Fig. 4(B) (left)). Interestingly, bioparticles concentrate at one target location and another in an alternate manner (Fig. 4(B) (right)). The dynamic behavior observed here may be used to contentiously explore the search space and to deal with a dynamically changing
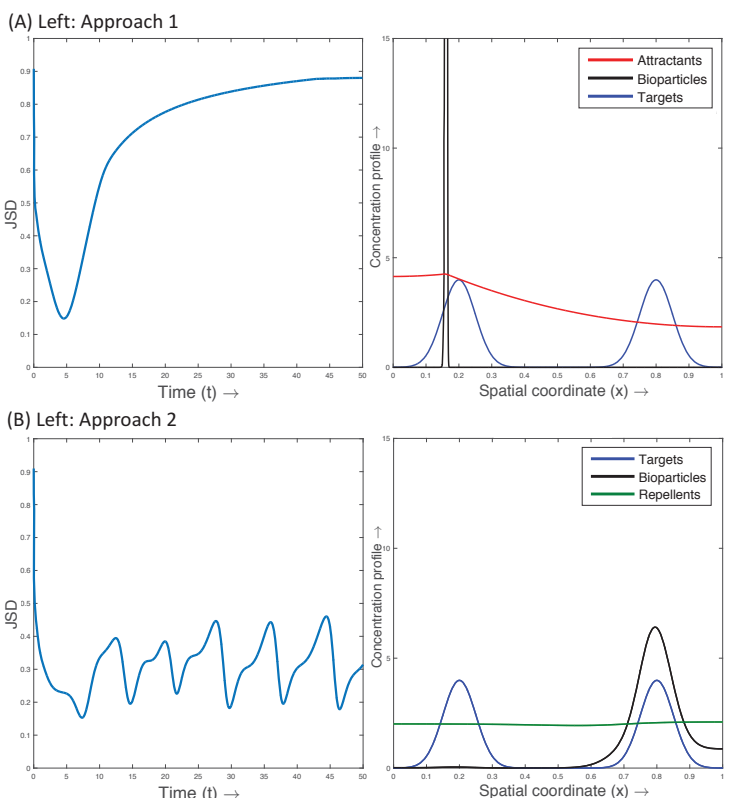

(C) Left: Approach 3
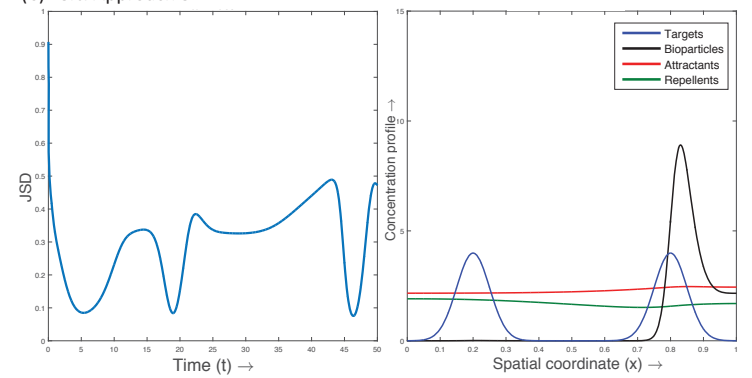

Figure 4: Experimental results. Bioparticles are injected at the left corner.

target distribution.

When Approach 3 is used, JSD and the bioparticle distribution change in a chaotic manner (Fig. 4(C) (left) and (right)). This result shows that a highly complex behavior of bioparticles can emerge when both attractants and repellents are used.

\subsubsection{Performance Optimization}

In performance optimization, we reserve $V_{a}$ and $V_{r}$ to be controllable. $V_{a}$ and $V_{r}$ represent the sensitivity of bioparticles to attractants and repellents, and the two parameters may be tuned in development of bioparticles. For example, when bioparticles are developed from bacterial cells, bacteria may be genetically engineered to change the expression level of cell surface receptors for attractants and repellents to control their sensitivity to the two types of molecule.

Fig. 5 shows the averaged JSD value computed from time $t=10$ to 50 as the performance indicator. In Fig. 5(A), Approach 1 is used and $V_{a}$ is regulated between 0 and 2. In Fig. 5(B), Approach 2 is used and $V_{r}$ is regulated between 0 and 2. In Fig. 5(C) and (D), Approach 3 is used where $V_{a}$ is regulated with $V_{r}=0.1$ in Fig. $5(\mathrm{C})$ or where $V_{r}$ is regulated with $V_{a}=0.01$ in Fig. 5(D). In each of Fig. 5(A)(D), averaged JSD values are obtained with the two initial 
(A) Approach 1

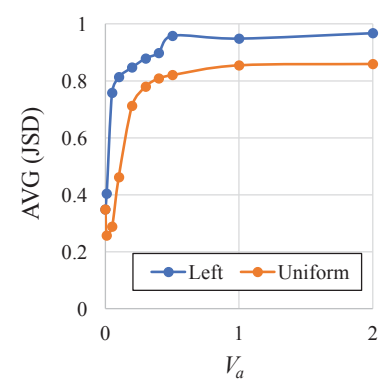

(C) Approach 3

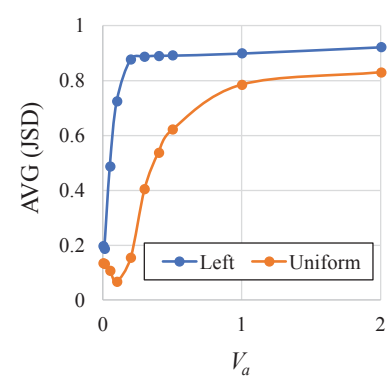

(B) Approach 2

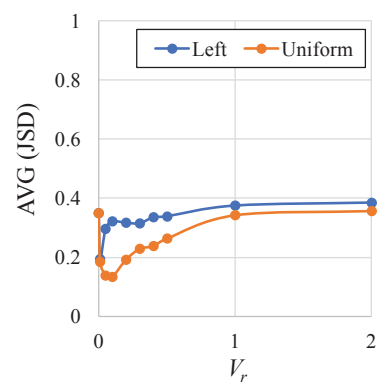

(D) Approach 3

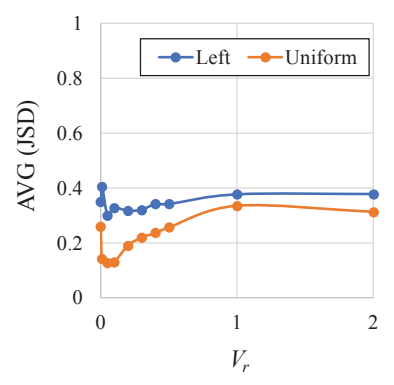

Figure 5: Performance optimization

conditions: "uniform" meaning that bioparticles are injected uniformly in the interval and "left" meaning that bioparticles are injected at the left corner of the interval.

When Approach 1 is used, the averaged JSD quickly increases as $V_{a}$ increases (Fig. 5(A)) in both cases of "uniform" and "left". In both cases, JSD degrades quickly, since bioparticles over-concentrate more around the target locations as $V_{a}$ increases.

When Approach 2 is used, the averaged JSD also increases as $V_{r}$ increases (Fig. 5(B)) in both cases of "uniform" and "left". In comparison to Approach 1, JSD in Approach 2 increases more slowly with $V_{r}$ and it is bounded by the lower value (around 0.4). This indicates that Approach 2 is easier to tune than Approach 1 and more practical to obtain better performance. In Approach 2, for all $V_{r}$ values tested, we observe oscillatory behavior in JSD (data not shown).

When Approach 3 is used, the averaged JSD becomes smaller than those in Approach 1 (compare Fig. 5(A) and (C)) and Approach 2 (compare Fig. (B) and (D)) in both cases of "uniform" and "left". This demonstrates the effectiveness of Approach 3 where attractants and repellents both are used for multi-target detection and gravitation.

\section{CONCLUSION}

In this paper, we investigated the performance of selforganizing bioparticles for multi-target detection and gravitation problems. We developed three approaches (1) the attractant-based approach, (2) repellent-based approach, and (3) combined approach, and examined how bioparticles behave in each approach. We also explored parameter space to optimize the performance of each approach.

The major findings from the numerical experiments are the following: The attractant-based approach can allow biopar-

ticles to over-concentrate at a location in the environment; this may lead to an undesirable bioparticle distribution from the perspective of drug delivery applications. The bioparticle distribution can exhibit highly complex behavior when repellents are used. The combined approach with both attractants and repellents achieves the best performance among the three approaches. Further, the initial bioparticle distribution appears to affect the final bioparticle distribution and thus the performance.

To provide an effective solution for drug delivery, our future work considers realistic constraints and conditions such as intricate terrains found in the in-body environment and uniform dispersion of drug molecules in target areas, in addition to the presence of multi-targets. Our future work also includes developing biologically plausible models of bioparticles and performing feasibility studies through in vitro experiments.

\section{Acknowledgments}

This work was supported through the Osaka University $\mathrm{Hu}-$ manware Innovation Program, a Leading Graduate School Program by the Japan Society of the Promotion of Science.

\section{REFERENCES}

[1] E. Bonabeau, M. Dorigo, and G. Theraulaz. Swarm intelligence: from natural to artificial systems. Oxford University Press, 1999.

[2] E. O. Budrene and H. C. Berg. Dynamics of formation of symmetrical patterns by chemotactic bacteria. Nature, 376(6535):49-53, 1995.

[3] S. Iwasaki, J. Yang, A. O. Abraham, J. L. Hagad, T. Obuchi, and T. Nakano. Modeling multi-target detection and gravitation by intelligent self-organizing bioparticles. In IEEE Global Communications Conference (GLOBECOM 2016), 2016.

[4] M. Nagy, Z. Akos, D. Biro, and T. Vicsek. Hierarchicalgroupdynamics in pigeon flocks. Nature, 464(7290):890-893, 2010.

[5] T. Obuchi, Y. Okaie, T. Nakano, T. Hara, and S. Nishio. Inbody mobile bionanosensor networks through non-diffusion-based molecular communication. In Proc. 2015 IEEE Conference on Communications (ICC), pages 1078-1084, 2015.

[6] Y. Okaie, T. Nakano, T. Hara, and S. Nishio. Target Detection and Tracking by Bionanosensor Networks. SpringerBriefs in Computer Science. Springer, 2016.

[7] Y. Okaie, T. Nakano, T. Hara, T. Obuchi, K. Hosoda, Y. Hiraoka, and S. Nishio. Cooperative target tracking by a mobile bionanosensor network. IEEE Transactions on Nanobioscience, 13(3):267-277, 2014.

[8] J. K. Parrish, S. V. Viscido, and D. Grunbaum. Self-organized fish schools: An examination of emergent properties. Biol. Bull., 202(3):296-305, 2002.

[9] G. Wei, P. Bogdan, and R. Marculescu. Bumpy rides: Modeling the dynamics of chemotactic interacting bacteria. IEEE Journal of Selected Areas in Communication (JSAC), 31(12):879-890, 2013. 